Louis Leveuf • Libor Navrátil • Vincent Le Sauxi •

Yann Marco · Jérôme Olhagaray · Sylvain Leclercq

\title{
Constitutive equations for the cyclic behaviour of short carbon fibre-reinforced thermoplastics and identification on a uniaxial database
}

Received: 17 November 2017 / Accepted: 18 December 2017

(C) Springer-Verlag GmbH Germany, part of Springer Nature 2018

\begin{abstract}
A constitutive model for the cyclic behaviour of short carbon fibre-reinforced thermoplastics for aeronautical applications is proposed. First, an extended experimental database is generated in order to highlight the specificities of the studied material. This database is composed of complex tests and is used to design a relevant constitutive model able to capture the cyclic behaviour of the material. A general 3D formulation of the model is then proposed, and an identification strategy is defined to identify its parameters. Finally, a validation of the identification is performed by challenging the prediction of the model to the tests that were not used for the identification. An excellent agreement between the numerical results and the experimental data is observed revealing the capabilities of the model.
\end{abstract}

Keywords Short fibre-reinforced thermoplastic - Cyclic loading - Constitutive equations - Complex experimental database $\cdot$ Thermodynamics of irreversible processes

\section{Introduction}

The mass reduction problematic has always been of primary interest in the aeronautical industry. The continuous development of composites manufacturing and scientific investigations since the 1980s lead these materials to be predominant (in mass) over metallic materials. For example, the Airbus A350 XWB uses 53\% of composites materials. The large majority of these composite materials are thermosets reinforced with continuous fibres. In order to go beyond the $50 \%$ proportion, other parts have to be replaced. The aeronautical industry is now

Communicated by Johlitz, Laiarinandrasana and Marco.

L. Leveuf · L. Navrátil · V. Le Saux ( $\varangle)$. Y. Marco

ENSTA Bretagne, Institut de Recherche Dupuy de Lôme (iRDL), FRE CNRS 3744, Brest, France

E-mail: vincent.le_saux@ensta-bretagne.fr

L. Leveuf

E-mail: louis.leveuf@ensta-bretagne.org

L. Navrátil

E-mail: libor.navratil@ensta-bretagne.org

J. Olhagaray

Safran Composites, Itteville, France

E-mail: jerome.olhagaray@safrangroup.com

S. Leclercq

Safran Landing Systems, Vélizy, France

E-mail: sylvain.leclercq@safrangroup.com 
considering the use of the short fibre-reinforced thermoplastics. The main reasons are the compatibility of these materials with the injection moulding process which allows both a cost reduction and the definition of complex parts with stiffeners and ribs to circumvent the loss in rigidity compared to metallic solutions.

Under service conditions, these parts are likely to undergo cyclic loadings with cyclic climatic variations (temperature and/or humidity) and must therefore be correctly designed against high cycle fatigue under complex thermo-hygro-mechanical loadings. This is usually achieved throughout a two-step process $[3,19]$. First, a thermomechanical model is needed to accurately capture the cyclic behaviour of the material [2,7, $10,13,17,19,29,30]$. Then, based on this stabilized cyclic state, an end-of-life fatigue criterion needs to be established in order to predict the fatigue life $[8,14,16,18,27]$. In this paper, we focus only on the constitutive model and the main objective is to propose a model able to describe the stabilized cyclic thermomechanical behaviour.

Describing accurately the cyclic behaviour of short fibre-reinforced thermoplastics is a very difficult task for two main reasons. The first is related to the injection moulding process which leads to complex fibre orientations and thus a strong anisotropy $[4,9,33]$. These orientations can be predicted by simulating the injection process which provides the results as a second-order orientation tensor [1]. The results of these simulations can be taken into account in the mechanical model using two different approaches: micromechanical models [15,31] or phenomenological approaches $[2,19]$. Although the first category is appealing, its application when nonlinear phenomena need to be taken into account remains a very difficult task and its computation time is still an issue for finite elements computations on complex part. The second is related to the influence of the environment on the thermomechanical response of the composite [20]. The cyclic behaviour needs to remain valid over a very wide range of experimental conditions, leading to extensive experimental database.

We focus in this paper on the definition of a constitutive model that relies on the thermodynamics of irreversible processes. In order to be able to justify each constitutive element of the model, we focus here on isothermal experiments performed for a single mean orientation, i.e. the model will be isotropic. The extension to anisotropy will be discussed in a forthcoming paper. The paper is divided into 5 sections. In Sect. 2, some details on the materials as well as on the experimental set-up are given. In Sect. 3, the experimental results are presented and each basic element of the forthcoming model is introduced and discussed. In Sect. 4, the 3D model is presented within the thermodynamics of irreversible processes and a specific 1D formulation is then proposed to ease the identification of the model. In Sect. 5, the identification of the parameters is achieved and the capability of the model is discussed throughout the comparison to complex tests outside the identification database. Finally, the strength and limits of the model are summarized in the conclusion.

\section{Material and specimen and experimental set-up}

\subsection{Material}

The material investigated is a thermoplastic polymer matrix (PEEK) reinforced with $30 \%$ (in weight) of short carbon fibres. The mean diameter and length of the fibres are below 10 and $200 \mu \mathrm{m}$, respectively. The samples are milled from $4 \mathrm{~mm}$ thickness injection moulded plates along the injection direction as illustrated in Fig. 1. The plates are injected using the regular process parameters advised by the material supplier. A micrography of the microstructure obtained on the side of the specimen is shown in Fig. 1. Strong variations of the fibres' orientation along the thickness can be noticed due to the well-known "skin-core" effect induced by the injection moulding process. Indeed, the fast cooling of the material on the mould surfaces generates a strong orientation of the fibres along the viscous flow direction near the walls (fibres appear vertical here) and a nearly orthogonal orientation at the core (fibres are normal to the paper plane and appear as circles). As a consequence, the microstructure of these samples is complex and they can already be considered as structures.

In order to avoid any structural effects related to the gradient of fibres' orientation, thin specimens of $1 \mathrm{~mm}$ thickness, corresponding to the skin of the full specimen, are extracted from the thick one $(4 \mathrm{~mm}$ thickness, see Fig. 1) using a milling operation in order to obtain a "homogeneous" microstructure so that the mechanical behaviour of the material is evaluated for a better mastered microstructure. In order to avoid any variation of the microstructure from one specimen to another, only one sample per plate was used and all the samples were milled from the central location of the plates. Specific precautions were taken during the generation of these sample in order to get a repeatable thickness, thus microstructure, for all the samples considered. The possible residual stresses induced by the process need to be discussed. The evaluation of these stresses is clearly difficult. In the case investigated here, we neglected these stresses on the basis of two observations. The first one is that the samples came perfectly straight and planar after the milling 


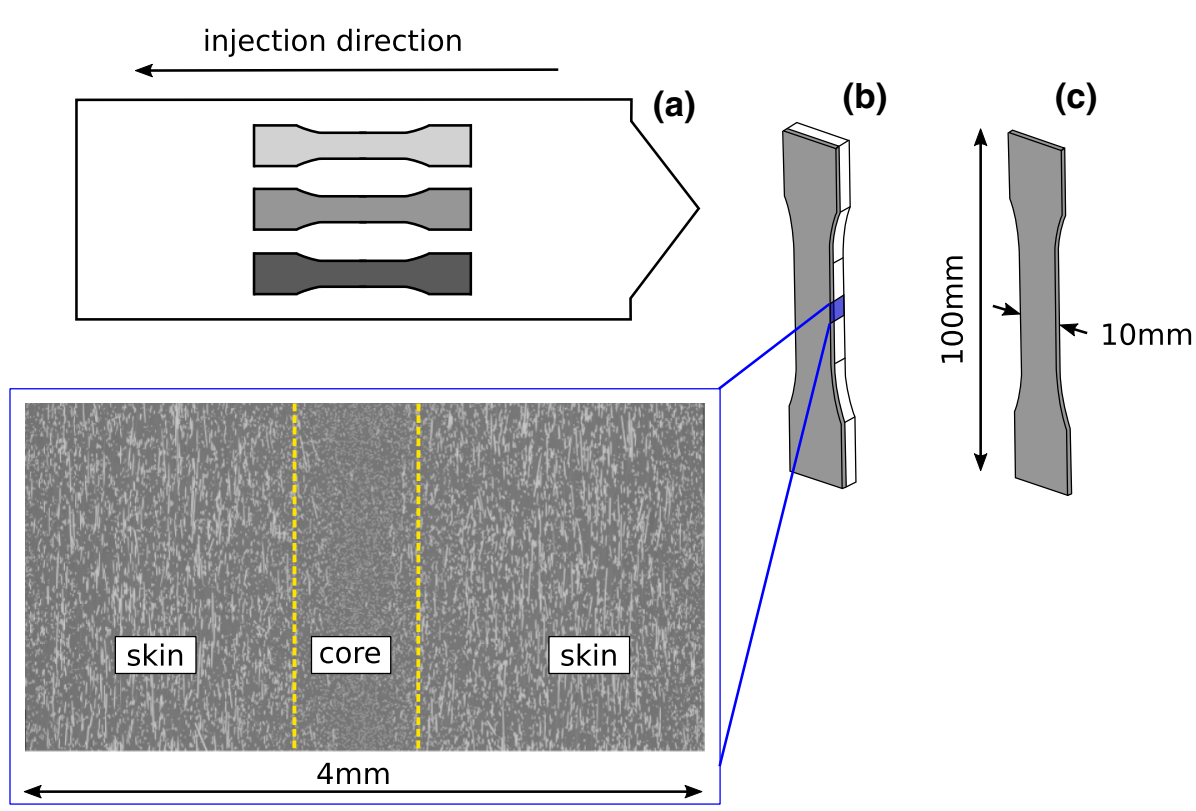

Fig. 1 Skin specimen extracted from the full specimen and corresponding microstructure (a). Only the medium grey sample a is considered, and one skin is extracted $(\mathbf{b}, \mathbf{c})$

operation from the injected plates. The residual stresses, if any, therefore seemed balanced over the length and the thickness of the samples. The second observation is that the $1 \mathrm{~mm}$ thick sample is perfectly planar. Moreover, during the polishing steps leading from the $4 \mathrm{~mm}$ thick sample to the $1 \mathrm{~mm}$ thick one, no visible bending was observed. It therefore seems that the internal stress gradient was limited in the original sample.

\subsection{Experimental set-up}

All the tests are performed on an electrodynamical INSTRON testing machine (model E10000) at room temperature. Local stress is measured thanks to a $10 \mathrm{kN}$ force cell, and the local strain was measured using the digital image correlation technique. A QImaging Retiga 6000 camera has been used to capture the images. The camera is equipped with a $16 \mathrm{~mm}$ diagonal sensor with a maximum resolution of 6.05 Mpixels and a pitch of $4.54 \mu \mathrm{m}$. A Nikon ED AF Micro Nikkor 70-180 mm 1:4.5-5.6D lens has been associated with the camera in order to analyse with high accuracy a small area of the specimen corresponding to the gauge length. All the post-processing (strain computed from a spatial mean over the gauge length for every image) has been performed using the NCorr software [5]. A dedicated system has been developed to synchronize all the data (images, force, global displacement) based on a homemade LabView software in order to ease the post-processing.

\section{Experimental observations}

This section is dedicated to the experimental database. For confidentiality issues, all the energetic and mechanical data are divided by arbitrary values, kept the same for all the tests to allow the comparison. These data are meant to highlight some specific characteristic features of the behaviour of the material and to be used for the forthcoming model identification and validation procedures. The idea is to define an experimental strategy requiring a limited number of experiments complex enough to highlight the specific aspects of the nonlinear behaviour of such materials, as already proposed by [19]. Figure 2 presents an overview of the tests performed. The test procedures are detailed in the forthcoming subsections. 


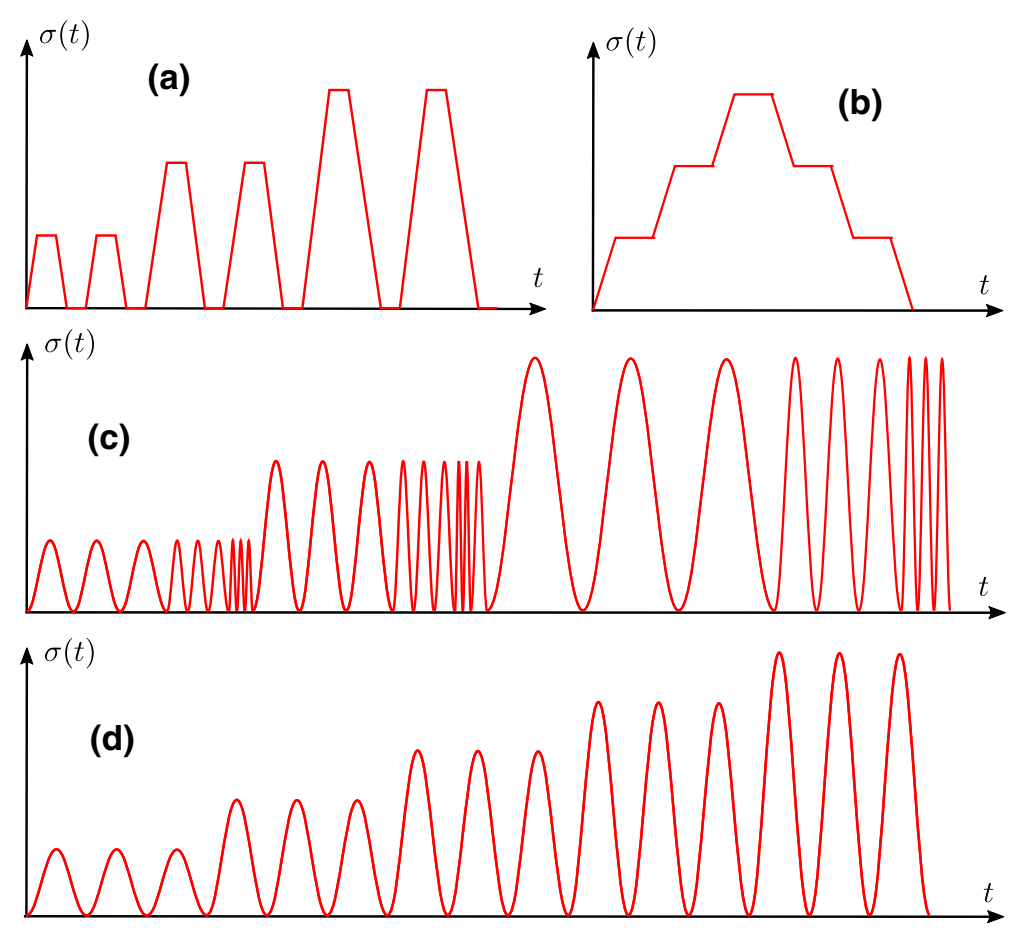

Fig. 2 Definition of the mechanical tests performed. All the tests have been load controlled and performed on tensile specimens (see Fig. 1). a CRR, b ANH, c CYC, d HB-up

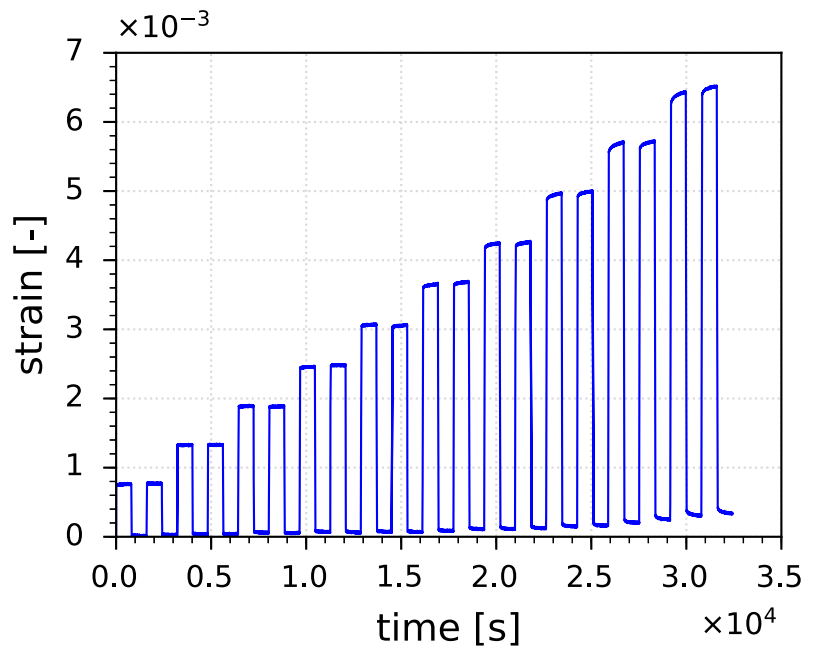

Fig. 3 Cyclic creep-recovery (CCR) test

\subsection{Cyclic creep-recovery (CCR) test}

The principle of the first type of experiment is presented in Fig. 2a. This test consists of incremental creep tests at various stress levels with a recovery period at the end of each creep level. The recovery period is meant to highlight any non-recoverable strain that may be considered as plastic strain. It should be pointed out that these irrecoverable strains may be recovered for a longer recovery period and that we will consider them as irrecoverable. It is therefore an appropriate way to detect any plastic threshold. The creep blocks are repeated two times in order to be able to separate the viscoelastic effects from the (visco)plastic ones, assuming that the plastic phenomena are not triggered during the second repetition.

The results are shown in Fig. 3. We can observe for the first loading block that the strain is almost recovered, which is no longer the case for the following loading blocks. Some plastic phenomena are therefore activated 


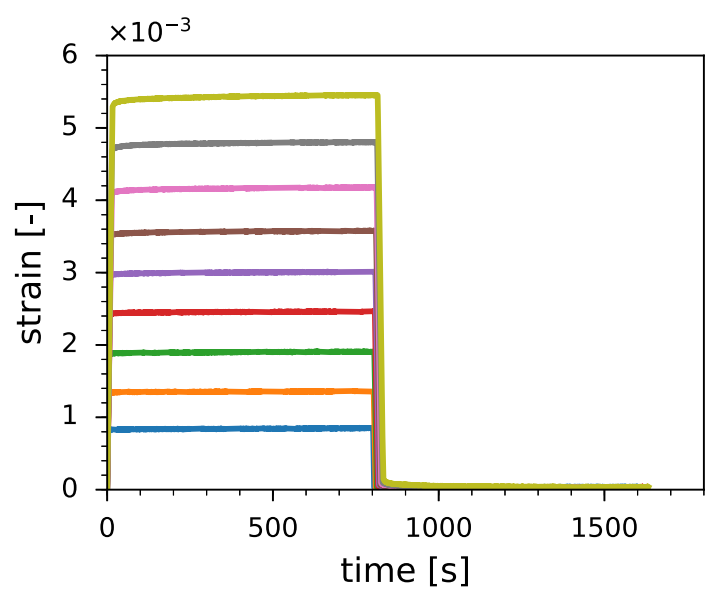

Fig. 4 Cyclic creep-recovery test without the plastic strains

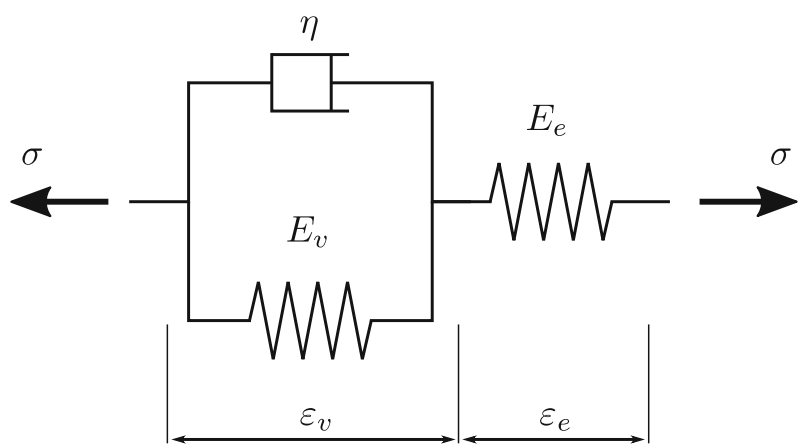

Fig. 5 1D rheological scheme used to interpret the CCR test

during the increasing stress blocks. During the decreasing stress blocks, the residual strains at the end of the recovering period do not evolve, meaning they are dependant on the maximum stress level reached during the test.

To investigate the viscoelastic phenomena, we propose to analyse the second block more in detail. Assuming that the total strain $\varepsilon$ can be divided into an elastic strain $\varepsilon_{\mathrm{e}}$, a viscoelastic strain $\varepsilon_{\mathrm{v}}$ and a plastic strain $\varepsilon_{\mathrm{p}}$ such as $\varepsilon=\varepsilon_{\mathrm{e}}+\varepsilon_{\mathrm{v}}+\varepsilon_{\mathrm{p}}$, we can remove the plastic strain assuming the plastic strains do not vary during the second block. This approach is possible since the viscoelastic strains seem to be stabilized at the end of the recovering period. Therefore, the plastic strain can be evaluated as the residual strain at the end of the first creep-recovery cycle of each block. The elastic-viscoelastic strains are then computed by subtracting the plastic strain from the total strain. The results are presented in Fig. 4. It should be underlined that the suggested approach seems relevant as the strain evaluated without the plastic contribution returns almost to zero at the end of the recovering period. Moreover, we also suppose that there is no history effect, meaning that in the forthcoming analysis, the step $n$ is not affected by the step $n-1$. This last hypothesis allows us to set the origin of the time at the beginning of each block (see Fig. 4).

We propose a simple model (Fig. 5) to describe the elastic and viscoelastic phenomena.

The model consists of an elastic branch (modulus $E_{\mathrm{e}}$ ) in series with a Poynting-Thomson branch (modulus $E_{\mathrm{v}}$, viscosity $\eta$ ). This $1 \mathrm{D}$ model is governed by the following differential equation

$$
\frac{E_{\mathrm{e}}+E_{\mathrm{v}}}{E_{\mathrm{e}}} \sigma+\frac{\eta}{E_{\mathrm{e}}} \dot{\sigma}=E_{\mathrm{v}} \varepsilon+\eta \dot{\varepsilon}
$$

The elastic modulus $E_{\mathrm{e}}$ is identified on the first loading (not shown in Fig. 4). The viscoelastic parameters ( $E_{\mathrm{v}}$ and $\eta$ ) are then identified for each stress level in order to see if constant values can fit the experimental data. The ratio $\tau=\eta / E_{\mathrm{v}}$ is evaluated on the recovering response since the evolution of the strain is governed by the equation

$$
\varepsilon(t)=\varepsilon_{R} \mathrm{e}^{-t / \tau}
$$




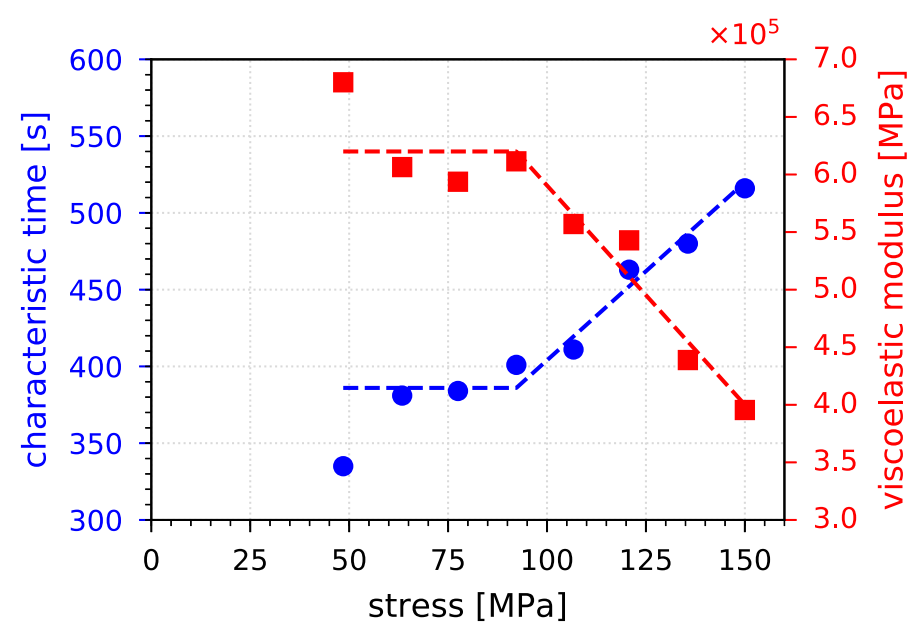

Fig. 6 Evolution of the characteristic time $\tau$ and modulus $E_{\mathrm{v}}$ as a function of the stress level. The viscosity $\eta$ can be deduced from this plot

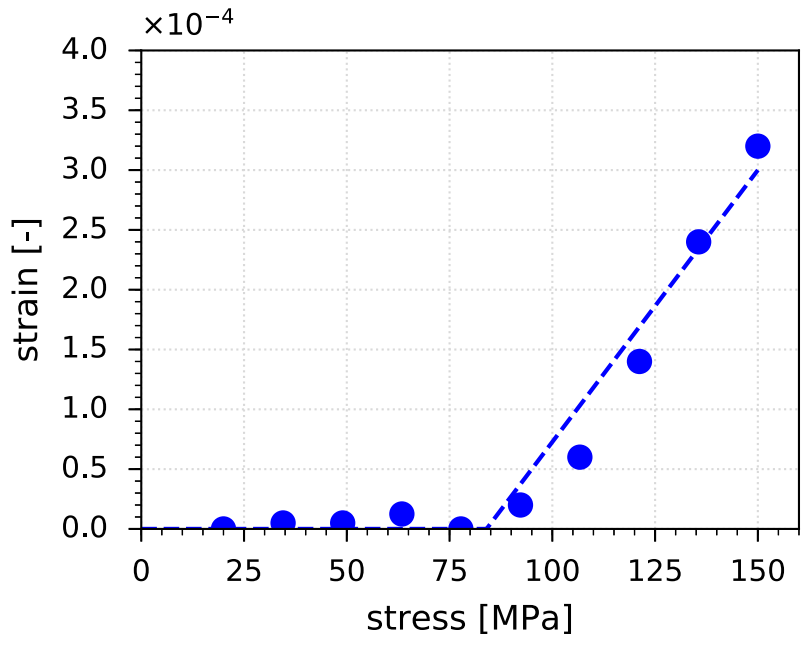

Fig. 7 Evolution of the residual strain as a function of stress

and the value of $\tau$ is identified using a simple fit of the quantity $\varepsilon(t) / \varepsilon_{R}$ where $\varepsilon_{R}$ is the strain at the very beginning of the recovering and can be evaluated graphically. The evolution of the viscoelastic modulus is computed based on the loading part of the block during which the strain can be expressed as follows

$$
\varepsilon(t)=\frac{E_{\mathrm{e}}+E_{\mathrm{v}}}{E_{\mathrm{e}} E_{\mathrm{v}}} \dot{\sigma}_{0} t-\frac{\eta \dot{\sigma}_{0}}{E_{\mathrm{v}}^{2}}\left(1-\mathrm{e}^{-E_{\mathrm{v}} t / \eta}\right)
$$

Therefore, for $t \rightarrow 0$

$$
\dot{\varepsilon}(t \rightarrow 0)=\frac{E_{\mathrm{e}}+E_{\mathrm{v}}}{E_{\mathrm{e}} E_{\mathrm{v}}} \dot{\sigma}_{0}
$$

The viscoelastic modulus can therefore be estimated using the initial slope of the strain during the first loading. Once $\tau$ and $E_{\mathrm{V}}$ are known, $\eta$ can be easily computed. The results are presented in Fig. 6 .

We can see that the characteristic times and viscoelastic modulus cannot be fitted by constant values. However, the viscosity can be considered as a constant for all the sequences of the CCR test (characterized here by the corresponding stress value). It should be underlined that this conclusion remains valid for the considered strain rate.

The residual strain at the end of each first loading cycle also provides valuable information regarding the nature and type of hardening. Figure 7 shows the evolution of the residual strain, that can be considered as plastic as a first approximation, as a function of the stress level of the corresponding step. A linear relationship 

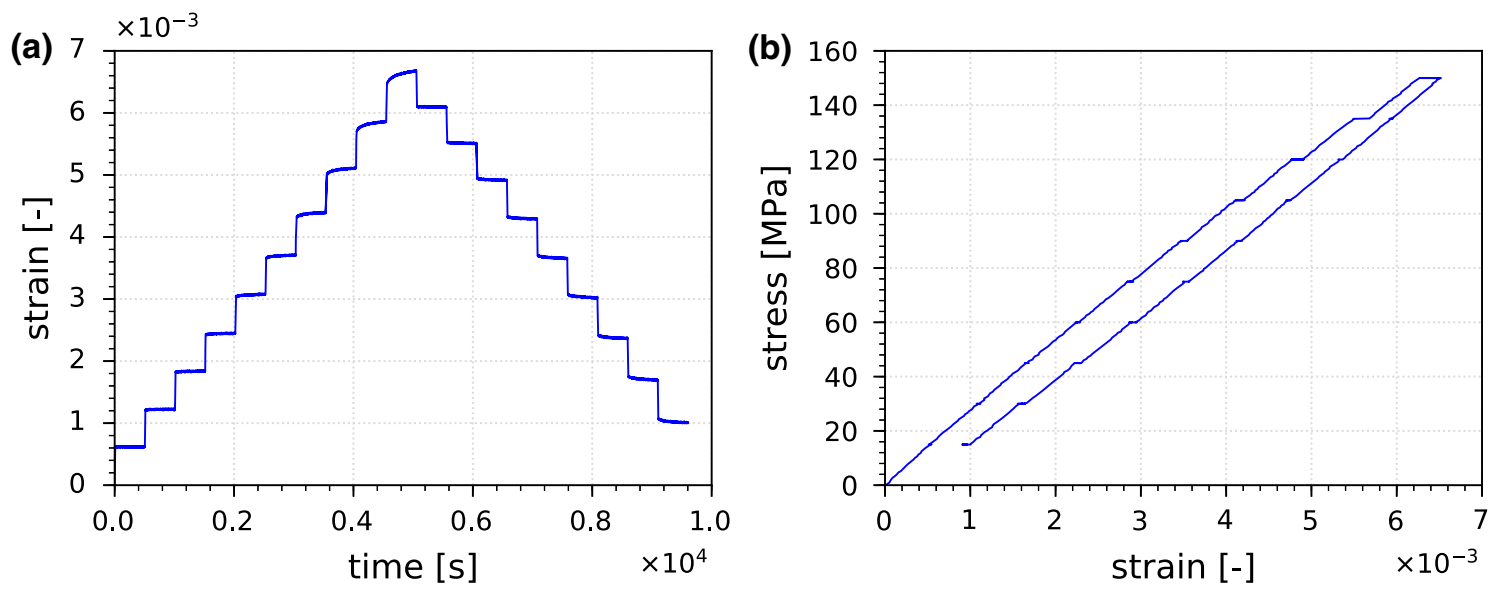

Fig. 8 Anhysteretic test

seems to provide a good approximation, meaning that a simple Prager kinematic hardening law is a good way to capture these plastic effects.

\subsection{Anhysteretic (ANH) test}

The anhysteretic test (see Fig. 2b) is a loading-unloading test with several creep periods at various stress levels, both during the loading and the unloading. This protocol provides a way to study nonlinear viscoelastic effects and to highlight any non-viscoelastic contribution to the hysteresis. In the case of a purely viscoelastic material, such test would allow a complete separation of the stress into a purely elastic stress (which leads to an elastic response) and a viscous stress (which leads to a hysteretic response), explaining why this kind to test is called "anhysteretic" test. The results are presented in Fig. 8. We can clearly point out a nonlinear viscoelasticity which appears to be plasticity dependant. Indeed, considering first the loading curve, the viscoelasticity behaviour increases with the plasticity evolution. The unloading part brings additional information. The first creep steps during the unloading phase reveal an almost elastic behaviour, and the viscoelasticity effects are visible only on the last steps, i.e. for the lowest stresses. This observation confirms that the viscoelasticity is actually not stress dependant but depends on a plastic property (that still needs to be defined). Moreover, and since the stress remains positive, this plastic behaviour is driven by, at least, a kinematic hardening (nothing can be deduced up to now regarding a isotropic hardening).

\subsection{Cyclic (CYC) test}

An accurate description of the hysteresis loop is mandatory as an energy-based criterion is considered for fatigue predictions. The last experiment is therefore a cyclic test at various frequencies ranging from 0.1 to $10 \mathrm{~Hz}$, investigating various short characteristic times for different stress levels (thus various strain rates). This test is also a convenient approach to study the evolution of the residual strains and highlight any ratcheting effects (see Fig. 9). In Fig. 9b, the fifth cycle obtained for the lowest frequency is plotted in order to reveal the stabilized cycle for various strain levels.

\subsection{Heat build-up (HB-up) test}

The last experiment is the so-called heat build-up test which consists of a set of cyclic tests at a given frequency $(2 \mathrm{~Hz})$ for increasing stress levels (Fig. 2d). This test is a good way to investigate the stress amplitude effect on the cyclic behaviour. During this test, a Flir Systems SC7600BB infrared camera was used to measure the temperature variation during the test. In order to increase the thermal resolution, a compensated pixelwise calibration was used [21]. The cyclic dissipation is evaluated from the temperature evolution using a methodology detailed elsewhere $[23,26,32]$. This test is a convenient way to challenge the energetic capabilities of the model that is crucial in order to use it in design loop against fatigue [12,24,32]. 

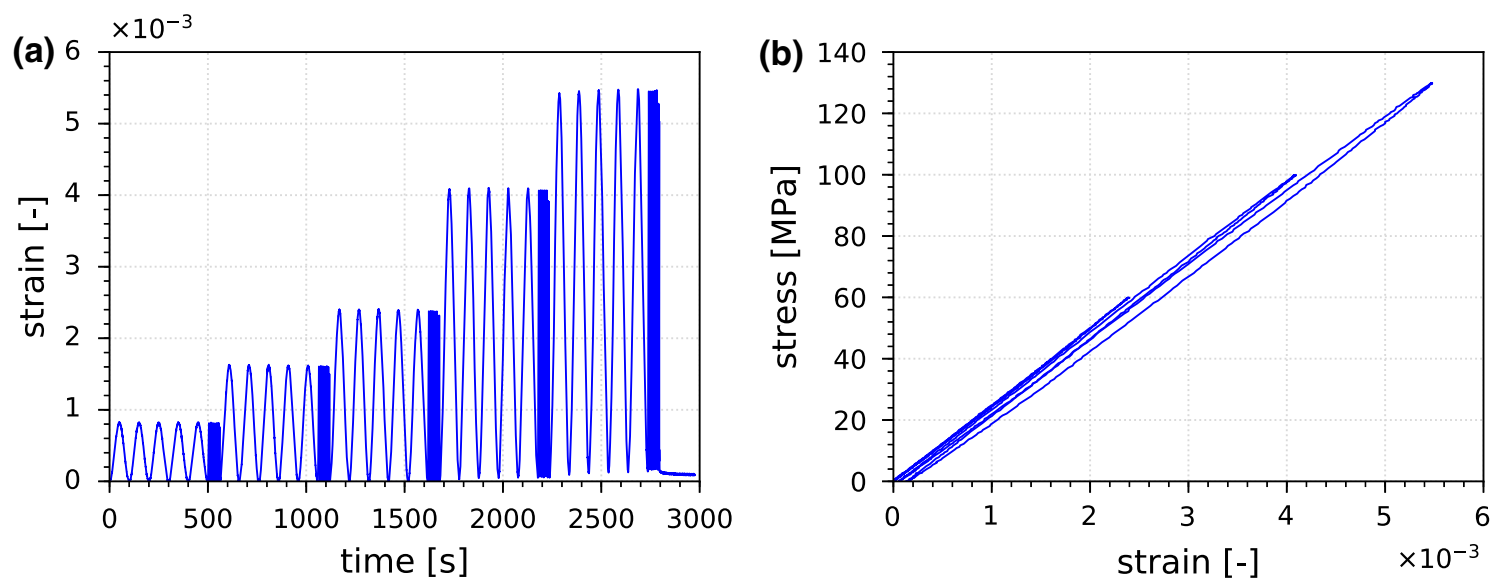

Fig. 9 Evolution of the strain for the cyclic test

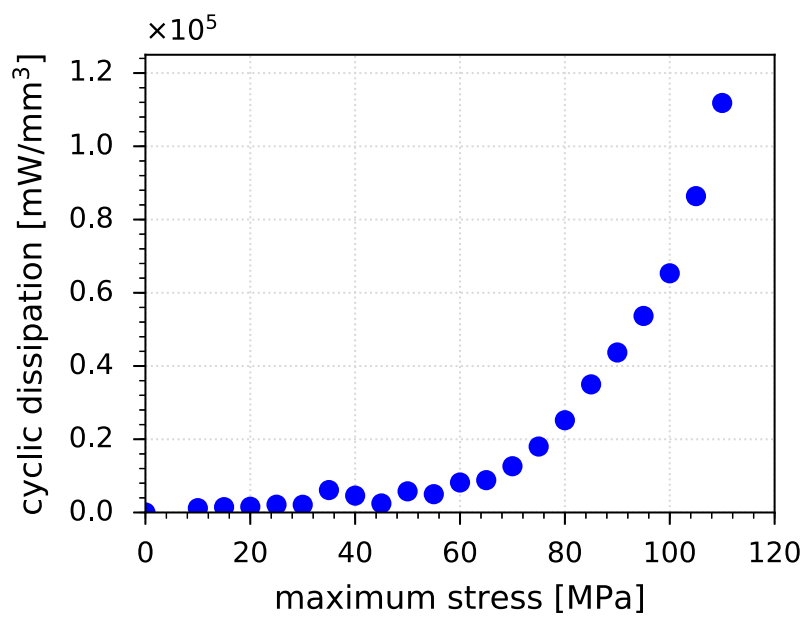

Fig. 10 Evolution of the cyclic dissipation as a function of the stress amplitude

Figure 10 presents the heat build-up curve obtained and highlights that the dissipation is evolving nonlinearly with the stress amplitude. For low stress amplitudes, the dissipation is close to zero and increases nonlinearly when the stress amplitude increases.

\section{Modelling}

The last section exhibits some specific features that must be described by the model. We propose in this section a general 3D formulation that will then be simplified into a $1 \mathrm{D}$ formulation in order to ease the identification process.

\subsection{General 3D formulation}

The proposed model lies within the framework of the generalized standard materials (GSM) defined by Halphen and Nguyen [11]. Four tensorial variables are needed to define the current mechanical state: the overall strain tensor $\boldsymbol{\varepsilon}$, associated with the stress tensor $\sigma$, the viscoelastic strain $\varepsilon_{\mathbf{v}}$, associated with the thermodynamical force $\mathcal{A}_{\mathbf{v}}$, the elasto-plastic strain $\boldsymbol{\varepsilon}_{\mathrm{p}}$, associated with the thermodynamic force $\mathcal{A}_{\mathrm{p}}$ and the hardening variable $\alpha$ associated with the centre of the plastic surface $\boldsymbol{X}$.

In the small perturbation framework, the overall strain can be splitted into

$$
\varepsilon=\varepsilon_{\mathrm{e}}+\varepsilon_{\mathrm{p}}+\varepsilon_{\mathrm{v}}
$$


where $\varepsilon_{\mathrm{e}}$ is the elastic strain (that shall not be considered as an internal variable). At the reference temperature, the Helmholtz free energy is the sum of the stored energies related to each modelled mechanism and depends on the state variables

$$
\rho \psi\left(\boldsymbol{\varepsilon}, \boldsymbol{\varepsilon}_{\mathbf{p}}, \boldsymbol{\varepsilon}_{\mathbf{v}}, \boldsymbol{\alpha}\right)=\frac{1}{2}\left(\boldsymbol{\varepsilon}-\boldsymbol{\varepsilon}_{\mathbf{p}}-\boldsymbol{\varepsilon}_{\mathbf{v}}\right): \mathbb{C}_{\mathrm{e}}:\left(\boldsymbol{\varepsilon}-\boldsymbol{\varepsilon}_{\mathbf{p}}-\boldsymbol{\varepsilon}_{\mathbf{v}}\right)+\frac{1}{3} C \boldsymbol{\alpha}: \boldsymbol{\alpha}+\frac{1}{2} \varepsilon_{\mathbf{v}}: \mathbb{C}_{\mathrm{v}}(\boldsymbol{\alpha}): \boldsymbol{\varepsilon}_{\mathbf{v}}
$$

where $\mathbb{C}_{\mathrm{e}}$ is the instantaneous elasticity tensor, $\mathbb{C}_{\mathrm{v}}$ is the viscoelasticity tensor and $C$ is a material constant. The thermodynamic forces can be evaluated from the partial derivative of the free energy with respect to the state variables

$$
\begin{aligned}
\boldsymbol{\sigma} & =\rho \frac{\partial \psi}{\partial \boldsymbol{\varepsilon}}=\mathbb{C}_{\mathrm{e}}:\left(\boldsymbol{\varepsilon}-\boldsymbol{\varepsilon}_{\mathbf{p}}-\boldsymbol{\varepsilon}_{\mathbf{v}}\right) \\
\boldsymbol{X} & =-\rho \frac{\partial \psi}{\partial \boldsymbol{\alpha}}=-\frac{2}{3} C \boldsymbol{\alpha}-\frac{\partial}{\partial \boldsymbol{\alpha}}\left[\frac{1}{2} \boldsymbol{\varepsilon}_{\mathbf{v}}: \mathbb{C}_{\mathrm{v}}(\boldsymbol{\alpha}): \boldsymbol{\varepsilon}_{\mathbf{v}}\right] \approx-\frac{2}{3} \boldsymbol{C} \boldsymbol{\alpha} \\
\mathcal{A}_{\mathbf{p}} & =-\rho \frac{\partial \psi}{\partial \boldsymbol{\varepsilon}_{\mathbf{p}}}=\boldsymbol{\sigma} \\
\mathcal{A}_{\mathbf{v}} & =-\rho \frac{\partial \psi}{\partial \boldsymbol{\varepsilon}_{\mathbf{v}}}=\boldsymbol{\sigma}-\mathbb{C}_{\mathrm{v}}(\boldsymbol{\alpha}): \boldsymbol{\varepsilon}_{\mathbf{v}}
\end{aligned}
$$

A demonstration of the simplification proposed in Eq. 8 is given in "Appendix A". This simplification is not meant to reduce the computational cost, but to simplify drastically the forthcoming evolution law related to $\dot{\alpha}$. To complete the description of the material behaviour, a dissipation potential $\varphi^{\star}$ convex and minimum in 0 (in order to lead a the thermodynamical consistency) needs to be defined. According to the approach suggested by [6], this potential is splitted into two contributions, namely the plastic contribution $\Omega_{p}$ and the viscous one $\Omega_{\mathrm{v}}$ such as

$$
\varphi^{\star}\left(\boldsymbol{\sigma}, \boldsymbol{X}, \boldsymbol{A}_{\mathbf{v}}\right)=\Omega_{\mathrm{p}}(\boldsymbol{\sigma}, \boldsymbol{X})+\Omega_{\mathrm{v}}\left(\boldsymbol{A}_{\mathbf{v}}\right)
$$

The plastic potential is

$$
\Omega_{\mathrm{p}}(\boldsymbol{\sigma}, \boldsymbol{X})=\mathcal{J}_{2}(\boldsymbol{S}-\boldsymbol{X})-\sigma_{y}+\frac{3}{4} \frac{\gamma}{C} \boldsymbol{X}: \boldsymbol{X}
$$

where $\mathcal{J}_{2}$ is the von Mises norm, $\boldsymbol{S}$ is the deviatoric part of $\boldsymbol{\sigma}$ and $\sigma_{y}$ is the yield stress. It should be underlined that, even though a linear Prager plasticity model seems sufficient to describe the plastic effects, we have chosen here to consider an Armstrong-Fredericks nonlinear kinematic hardening. The viscous potential is

$$
\Omega_{\mathrm{V}}\left(\boldsymbol{A}_{\mathbf{v}}\right)=\frac{1}{2} \mathcal{A}_{\mathbf{v}}: \mathbb{V}\left(\dot{\varepsilon}_{\mathbf{v}}\right)^{-1}: \mathcal{A}_{\mathbf{v}}
$$

where $\mathbb{V}$ is a fourth-order viscous tensor. The evolution laws are then deduced from these potentials

$$
\begin{gathered}
\dot{\boldsymbol{\varepsilon}}_{\mathrm{p}}=\dot{\lambda}_{\mathrm{p}} \frac{\partial \Omega_{\mathrm{p}}}{\partial \mathcal{A}_{\mathrm{p}}}+\dot{\lambda}_{\mathrm{v}} \frac{\partial \Omega_{\mathrm{v}}}{\partial \mathcal{A}_{\mathbf{p}}} \\
\dot{\boldsymbol{\alpha}}=\dot{\lambda}_{\mathrm{p}} \frac{\partial \Omega_{\mathrm{p}}}{\partial \boldsymbol{X}}+\dot{\lambda}_{\mathrm{v}} \frac{\partial \Omega_{\mathrm{v}}}{\partial \boldsymbol{X}} \\
\dot{\boldsymbol{\varepsilon}}_{\mathbf{v}}=\dot{\lambda}_{\mathrm{p}} \frac{\partial \Omega_{\mathrm{p}}}{\partial \mathcal{A}_{\mathbf{v}}}+\dot{\lambda}_{\mathrm{v}} \frac{\partial \Omega_{\mathrm{v}}}{\partial \mathcal{A}_{\mathbf{v}}}
\end{gathered}
$$

where $\dot{\lambda}_{\mathrm{p}}$ and $\dot{\lambda}_{\mathrm{v}}$ are Lagrange multipliers. In case of rate independent plasticity, the plastic multiplier $\dot{\lambda}_{\mathrm{p}}$ is determined by the consistency condition of plastic flow $f=\dot{f}=0$ which lead to $\dot{\lambda}_{\mathrm{p}}=\dot{p}$ [22] where $p$ is the equivalent plastic strain

$$
p=\sqrt{\frac{2}{3} \varepsilon_{\mathbf{p}}: \varepsilon_{\mathbf{p}}}
$$


The viscous multiplier $\dot{\lambda}_{\mathrm{v}}$ will be taken equal to unity [6]. We have

$$
\begin{aligned}
\dot{\boldsymbol{\varepsilon}}_{\mathbf{p}} & =\frac{3}{2} \dot{p} \frac{\mathbb{P}:(\boldsymbol{\sigma}-\boldsymbol{X})}{\mathcal{J}_{2}(\boldsymbol{S}-\boldsymbol{X})} \\
\dot{\alpha} & =\frac{3}{2} \frac{\gamma}{C} \dot{p} \boldsymbol{X}-\dot{\boldsymbol{\varepsilon}}_{\mathbf{p}} \\
\dot{\boldsymbol{\varepsilon}}_{\mathbf{v}} & =\mathbb{V}\left(\dot{\boldsymbol{\varepsilon}}_{\mathbf{v}}\right)^{-1}: \mathcal{A}_{\mathbf{v}}
\end{aligned}
$$

$\mathbb{P}$ is the fourth-order deviatoric projector. The fourth-order viscous tensor will be considered as isotropic and deviatoric leading to

$$
\dot{\boldsymbol{\varepsilon}}_{\mathbf{v}}=\frac{3}{2 \eta\left(\dot{\boldsymbol{\varepsilon}}_{\mathbf{v}}\right)} \operatorname{dev}\left(\mathcal{A}_{\mathbf{v}}\right)
$$

where $\eta$ is the viscosity. In order to capture the frequency effect under cyclic loadings, we use a nonlinear viscosity law based on the Ostwald-deWaele model $[25,28]$

$$
\eta\left(\dot{\boldsymbol{\varepsilon}}_{\mathbf{v}}\right)=K \mathcal{J}_{2}\left(\dot{\boldsymbol{\varepsilon}}_{\mathbf{v}}\right)^{n-1}
$$

with $n<1$ (meaning that the suggested law is related to a rheofluidifiant model) and

$$
\mathcal{J}_{2}\left(\dot{\varepsilon}_{\mathbf{v}}\right)=\sqrt{\frac{3}{2} \dot{\varepsilon}_{\mathbf{v}}: \mathbb{P}: \dot{\varepsilon}_{\mathbf{v}}}
$$

\subsection{D formulation}

All the experimental tests are force controlled, along single tensile direction and performed on tensile specimen. The stress tensor in the gauge length is therefore uniaxial and can be written as

$$
\boldsymbol{\sigma}(t)=\sigma(t) \mathbf{e}_{1} \otimes \mathbf{e}_{1}
$$

Since $\boldsymbol{X}$ and $\boldsymbol{\alpha}$ are deviatoric tensors, we have

$$
\begin{aligned}
& X(t)=X(t) \mathbf{e}_{1} \otimes \mathbf{e}_{1}-\frac{X(t)}{2}\left(\mathbf{e}_{2} \otimes \mathbf{e}_{2}+\mathbf{e}_{3} \otimes \mathbf{e}_{3}\right) \\
& \boldsymbol{\alpha}(t)=\alpha(t) \mathbf{e}_{1} \otimes \mathbf{e}_{1}-\frac{\alpha(t)}{2}\left(\mathbf{e}_{2} \otimes \mathbf{e}_{2}+\mathbf{e}_{3} \otimes \mathbf{e}_{3}\right)
\end{aligned}
$$

In the same manner, as the viscous strain tensor is supposed to be deviatoric, we have

$$
\boldsymbol{\varepsilon}_{\mathbf{v}}(t)=\varepsilon_{\mathrm{V}}(t) \mathbf{e}_{1} \otimes \mathbf{e}_{1}-\frac{\varepsilon_{\mathrm{v}}(t)}{2}\left(\mathbf{e}_{2} \otimes \mathbf{e}_{2}+\mathbf{e}_{3} \otimes \mathbf{e}_{3}\right)
$$

The 3D formulation of the model then reduced to

$$
\begin{aligned}
\sigma & =E_{\mathrm{e}}\left(\varepsilon-\varepsilon_{\mathrm{p}}-\varepsilon_{\mathrm{v}}\right) \\
X & =-\frac{2}{3} C \alpha \\
\dot{\varepsilon_{\mathrm{p}}} & = \begin{cases}0 & \text { if } f<0 \\
\frac{\dot{\sigma}}{C-\gamma X \operatorname{sign}(\sigma-X)} & \text { if } f=0\end{cases} \\
\dot{\varepsilon}_{\mathrm{v}} & =\frac{1}{K\left|\dot{\varepsilon}_{\mathrm{v}}\right|^{n-1}\left(\sigma-E_{\mathrm{v}}(\alpha) \varepsilon_{\mathrm{v}}\right)} \\
E_{\mathrm{v}} & =\sum_{i=0}^{k} a_{i} \alpha^{i} \\
f & =|\sigma-X|-\sigma_{y}
\end{aligned}
$$

The parameter $k$ introduced in Eq. 32 will be considered here equal to 1. Figure 11 proposes a rheological representation of the $1 \mathrm{D}$ model. 


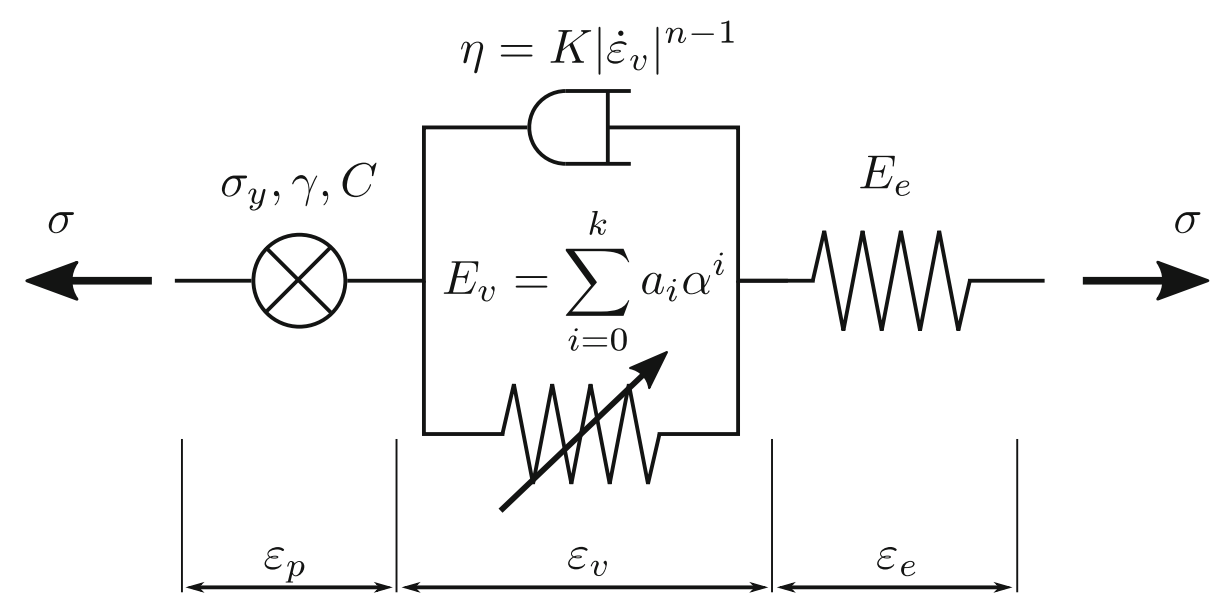

Fig. 11 1D rheological scheme of the proposed model

\section{Identification and validation}

In this section, we develop the identification strategy, as well as the validation procedure.

\subsection{Identification strategy}

An appropriate identification strategy is almost as important as the constitutive model. A total of 8 parameters need to be identified (see Fig. 11):

- elastic branch: $E_{\mathrm{e}}$;

- viscoelastic branch: $a_{i}, i \in[0,1], K$ and $n$;

- plastic branch: $\sigma_{y}, \gamma$ and $C$.

The approach suggested here aims at being efficient and requires only one experiment, namely the CCR test. As the test is stress controlled, these parameters are identified by minimizing the difference between the measured strain and the computed one. Due to the highly nonlinear aspect of the model and the coupling between the viscoelasticity and the plasticity, a sequential iterative approach is proposed here:

Sequence 1 the elastic block (Young's modulus $E_{\mathrm{e}}$ ) is first identified on the first loading assuming the stresses are too low for the plasticity to occur and the viscous effects lack time to express;

Sequence 2 the plastic block (yield stress $\sigma_{y}$ and the parameters of the kinematic hardening $C$ and $\gamma$ ) is then identified based on the residual strains at the end of the first recovering step during the loading (cf. Sect. 3.1 and Fig. 7);

Sequence 3 the viscoelastic branch $\left(K, n, a_{i}\right)$ is finally identified based on the full CCR test assuming the elastic and plastic strains are known.

Since the results of the sequence 3 are dependant on the ones of sequences 1 and 2, an iterative sequential loop is performed using an optimization algorithm until the convergence is reached. Figures 12, 13 and 14 present the results once the identification is over, and Table 1 presents the parameters. Based on this set of parameters, it becomes possible to numerically check the relevancy of the simplification made earlier (Eq. 8). All the details are given in "Appendix B".

\subsection{Validation}

The validation stage consists in simulating the experiments of the database that have not been used during the identification, namely the ANH, CYC and HB-up tests. 


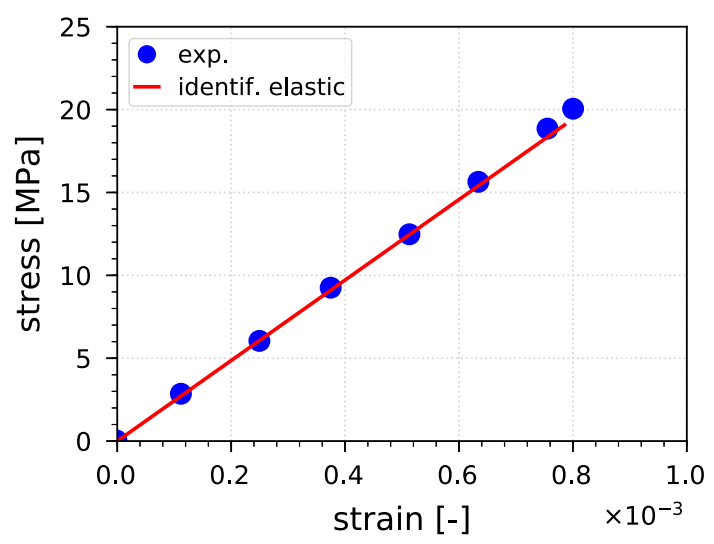

Fig. 12 Identification of the elastic parameter

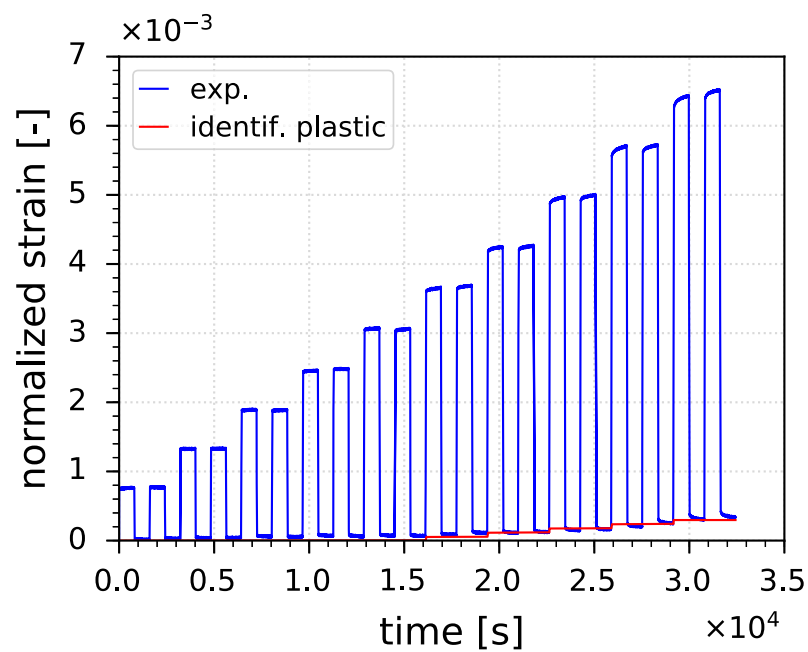

Fig. 13 Identification of the plastic parameters

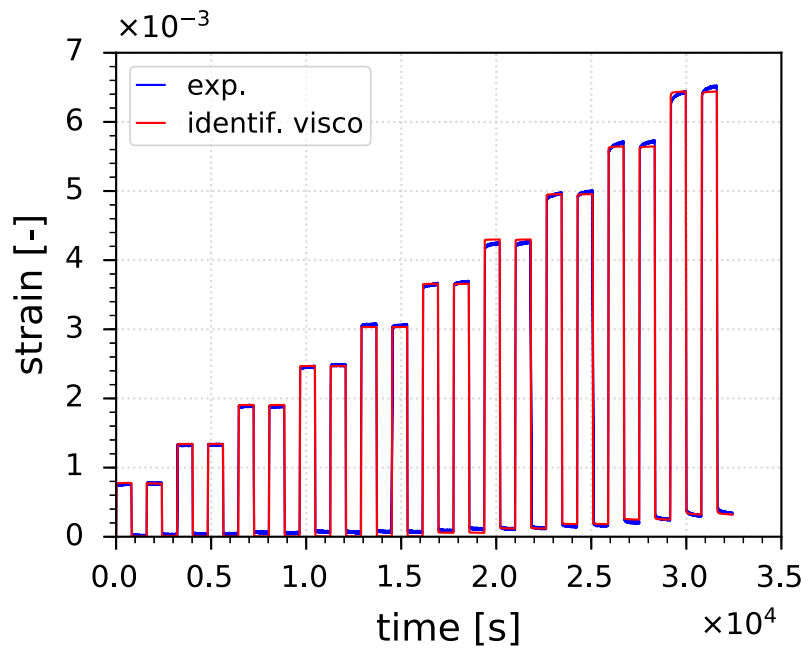

Fig. 14 Identification of the viscoelastic parameters 
Table 1 Identified parameters

\begin{tabular}{lc}
\hline Parameter & Value \\
\hline$E_{\mathrm{e}}$ & $26000 \mathrm{MPa}$ \\
$K$ & $400 \mathrm{MPa} \mathrm{s}^{2-n}$ \\
$n$ & 0.2 \\
$a_{0}$ & $1780320 \mathrm{MPa}$ \\
$a_{1}$ & $-19960 \mathrm{MPa}$ \\
$\sigma_{y}$ & $80 \mathrm{MPa}$ \\
$C$ & $234000 \mathrm{MPa}$ \\
$\gamma$ & 0 \\
\hline
\end{tabular}
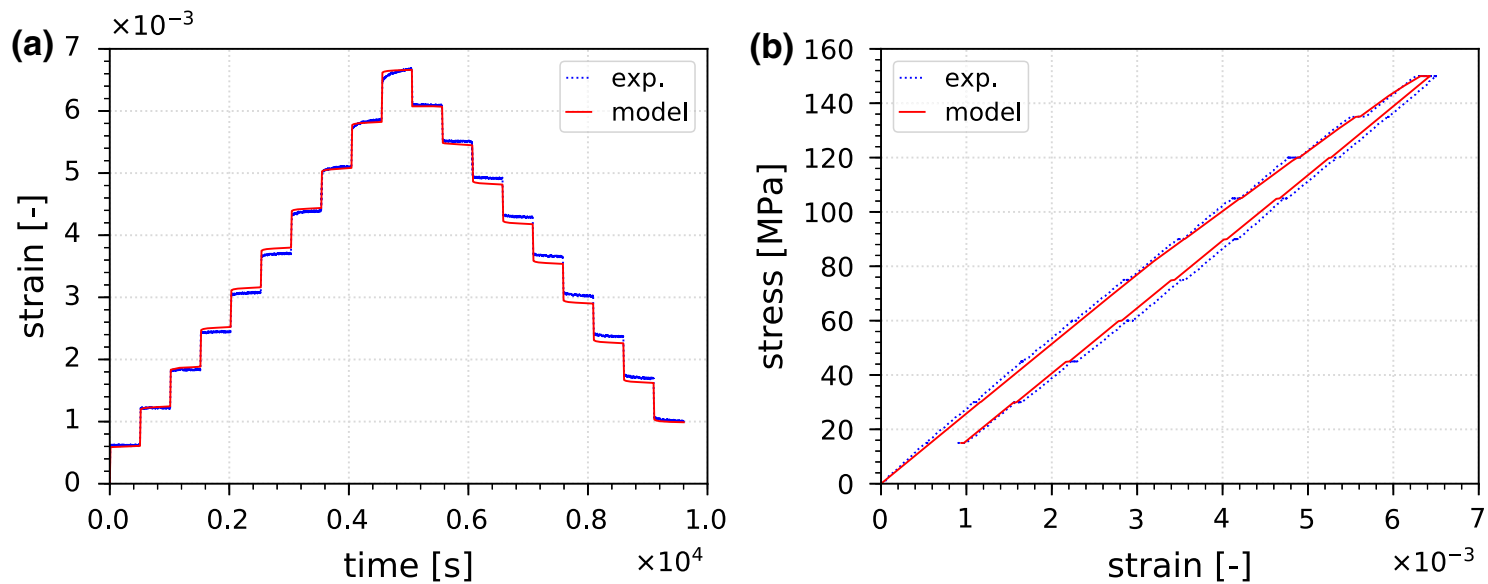

Fig. 15 Comparison between the prediction of the model and the experimental results for the anhysteretic test
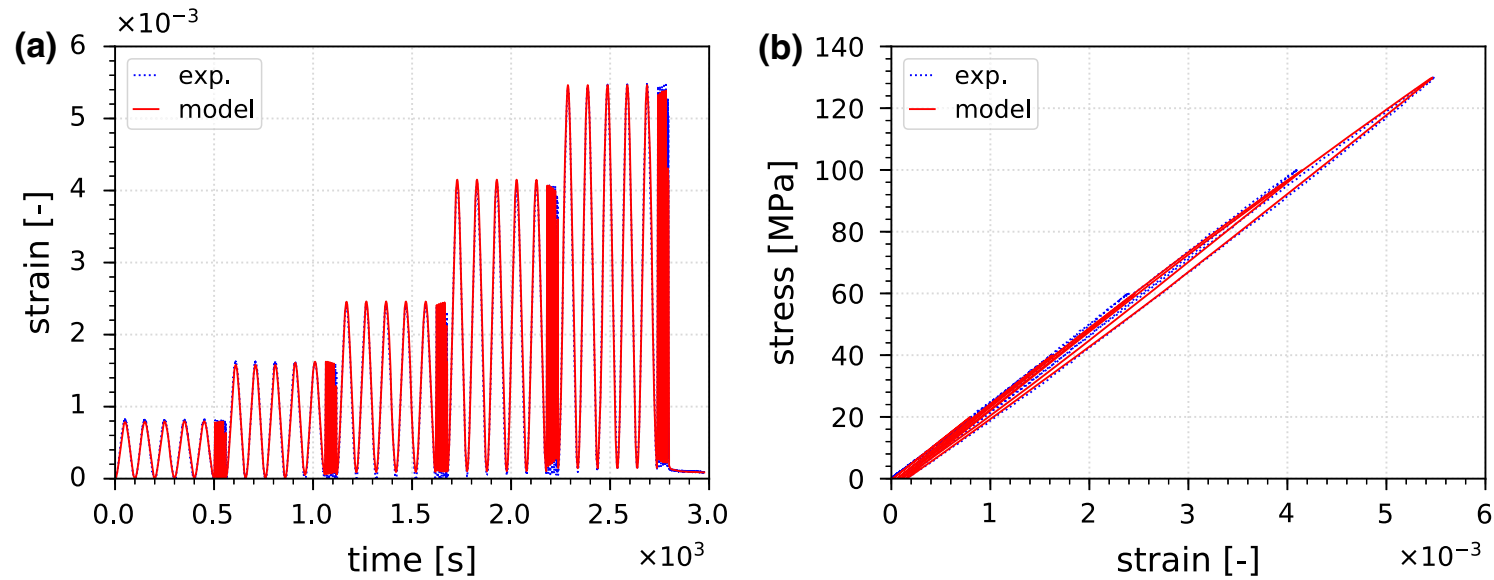

Fig. 16 Comparison between the prediction of the model and the experimental results for the cyclic test

\subsubsection{ANH test}

Figure 15 presents a comparison between the experimental results and the prediction of the model for the anhysteretic test, in terms of strain versus time (Fig. 15a) and stress versus strain (Fig. 15b). A very good agreement can be observed both during the loading and unloading phase of the test.

\subsubsection{CYC test}

Figure 16 presents a comparison between the experimental results and the prediction for the cyclic test, in terms of strain versus time (Fig. 16a) and stress versus strain for the stabilized cycle at the lowest frequency 


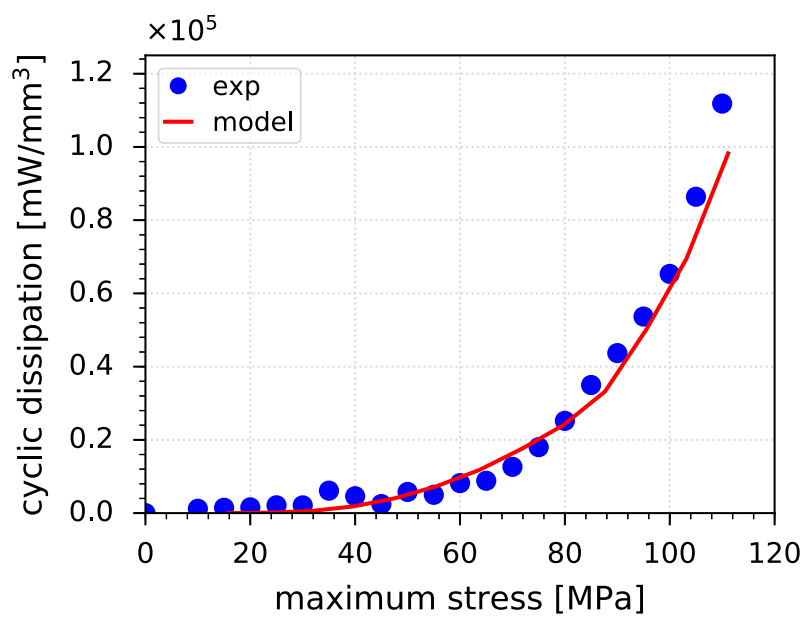

Fig. 17 Comparison between the cyclic dissipated evaluated from the model and measured

(Fig. 16b). Here again, the agreement is excellent, whatever the stress levels and the loading frequency, meaning that the model, and more specifically the nonlinear viscosity, is able to capture accurately the cyclic behaviour of the material.

\subsubsection{HB-up test}

The last validation test is the heat build-up test. We propose here to compare the experimental results to the prediction of the model in terms of cyclic dissipation. We therefore need to define the way to compute it with the model. Within the thermodynamics of irreversible processes framework, the instantaneous intrinsic dissipation can be evaluated thanks to the product of the internal variables with their corresponding thermodynamical forces [22]

$$
\mathcal{D}_{\mathcal{I}}=\sigma: \dot{\varepsilon}_{\mathbf{p}}-X: \dot{\alpha}-\mathcal{A}_{\mathbf{v}}: \dot{\varepsilon}_{\mathrm{v}}
$$

The cyclic intrinsic dissipation $\Delta_{c y c}$ can then be evaluated

$$
\Delta_{\mathrm{cyc}}=\frac{1}{T} \int_{t_{N}-T / 2}^{t_{N}+T / 2} \mathcal{D}_{\mathcal{I}}(t) \mathrm{d} t
$$

The evolution of the $\Delta_{\mathrm{cyc}}$ versus the number of cycles can then be plotted. It becomes then possible to compute the model cyclic dissipation by applying the same processing as the one applied to the experimental data (see Sect. 3.4). Figure 17 presents a comparison between the experimental and the computed cyclic dissipation. We can notice an excellent agreement between the data, meaning the model is able to capture very accurately the cyclic dissipation. The model seems therefore a very good candidate regarding fatigue design approaches based on an energy-based fatigue criterion.

\section{Conclusions}

A phenomenological constitutive model able to capture the cyclic response of short carbon fibres-reinforced thermoplastic has been detailed for a given fibres orientation tensor. The various modelling elements have been defined according to the experimental observations made on complex tests. A general 3D formulation relying on the thermodynamics of irreversible processes has been expressed. Then, a simplified 1D formulation has been written in order to ease the identification of the parameters. Finally, an efficient approach regarding the identification and validation of the parameters has been detailed. The model has shown its efficiency to describe accurately the various phenomena highlighted during the experiments, ranging from nonlinear viscosity for a wide range of strain rate to plasticity features and cyclic dissipation under cyclic loading. 
Acknowledgements The authors would like to thank C. Doudard, S. Calloch and S. Moyne from ENSTA Bretagne, R. Billardon from Safran Landing Systems and N. Carrère from Safran Composites for stimulating discussions and F. Montel from ENSTA Bretagne for the development of the LabView software. One of the authors (L. Leveuf) would like to thank Safran Composites for the funding of this study.

\section{A Simplification of the state equation relative to $X$}

The definition of a constitutive model that relies on the thermodynamics of irreversible processes requires some specific steps. One of them is the definition of the thermodynamical forces that can be defined as the derivative of the free energy with respect to the corresponding variable. Due to the coupling between the viscoelastic modulus to the plasticity, the definition of the backstress $\boldsymbol{X}$ is not standard and a simplification has been made in Sect. 4, Eq. 8. The goal of this appendix is to justify this simplification. Let's start by reminding the way to write the backstress $\boldsymbol{X}$

$$
\boldsymbol{X}=-\rho \frac{\partial \psi}{\partial \boldsymbol{\alpha}}=-\frac{2}{3} C \boldsymbol{\alpha}-\frac{\partial}{\partial \boldsymbol{\alpha}}\left[\frac{1}{2} \boldsymbol{\varepsilon}_{\mathbf{v}}: \mathbb{C}_{\mathrm{v}}(\boldsymbol{\alpha}): \boldsymbol{\varepsilon}_{\mathbf{v}}\right]
$$

The first term in this expression is classic, whereas the second one is specific to our model. Let us consider the expression

$$
\frac{\partial}{\partial \boldsymbol{\alpha}}\left[\frac{1}{2} \boldsymbol{\varepsilon}_{\mathbf{v}}: \mathbb{C}_{\mathbf{v}}(\boldsymbol{\alpha}): \boldsymbol{\varepsilon}_{\mathbf{v}}\right]
$$

Using an indicial notation and removing the ${ }_{v}$ subscript for clarity, this expression is equivalent to

$$
\frac{\partial}{\partial \alpha_{m n}}\left[\frac{1}{2} \varepsilon_{i j} C_{i j k l}(\boldsymbol{\alpha}) \varepsilon_{k l}\right]
$$

To ease this calculation, we first compute the term inside the bracket. Considering that $C_{i j k l}(\boldsymbol{\alpha})$ obeys to the Hooke law

$$
C_{i j k l}(\boldsymbol{\alpha})=\lambda(\boldsymbol{\alpha}) \delta_{i j} \delta_{k l}+\mu(\boldsymbol{\alpha})\left(\delta_{i k} \delta_{j l}+\delta_{i l} \delta_{j k}\right)
$$

with $\delta_{i j}$ being the Kronecker symbol, $\lambda(\boldsymbol{\alpha})$ and $\mu(\boldsymbol{\alpha})$ are the Lamé coefficients that can be written in terms of the Young modulus $E$ and the Poisson coefficient $v$ according to

$$
\lambda=\frac{E v}{(1+v)(1-2 v)},
$$

and

$$
\mu=\frac{E}{2(1+v)}
$$

Assuming that only the Young's modulus is dependant on the plasticity according to a simple polynomial function that relies on the $\mathcal{J}_{2}$ isotropic norm

$$
E=\sum_{m=0}^{p} a_{m} \mathcal{J}_{2}(\boldsymbol{\alpha})^{m}
$$

this implies that the Lamé coefficients can be written as

$$
\lambda(\boldsymbol{\alpha})=\frac{\sum_{m=0}^{p} a_{m} \mathcal{J}_{2}(\boldsymbol{\alpha})^{m} v}{(1+v)(1-2 v)}
$$

and

$$
\mu(\boldsymbol{\alpha})=\frac{\sum_{m=0}^{p} a_{m} \mathcal{J}_{2}(\boldsymbol{\alpha})^{m}}{2(1+v)}
$$


Combining Eqs. 39, 43 and 44 leads to

$$
\begin{aligned}
\varepsilon_{i j} C_{i j k l}(\boldsymbol{\alpha}) \varepsilon_{k l}= & \frac{\sum_{m=0}^{p} a_{m} \mathcal{J}_{2}(\boldsymbol{\alpha})^{m} v}{(1+v)(1-2 v)} \varepsilon_{i j} \delta_{i j} \delta_{k l} \varepsilon_{k l}+\frac{\sum_{m=0}^{p} a_{m} \mathcal{J}_{2}(\boldsymbol{\alpha})^{m}}{2(1+v)} \varepsilon_{i j} \delta_{i k} \delta_{j l} \varepsilon_{k l} \\
& +\frac{\sum_{m=0}^{p} a_{m} \mathcal{J}_{2}(\boldsymbol{\alpha})^{m}}{2(1+v)} \varepsilon_{i j} \delta_{i l} \delta_{j k} \varepsilon_{k l}
\end{aligned}
$$

Taking into account the property of the Kronecker symbol, this expression becomes

$$
\varepsilon_{i j} C_{i j k l}(\boldsymbol{\alpha}) \varepsilon_{k l}=\frac{\sum_{m=0}^{p} a_{m} \mathcal{J}_{2}(\boldsymbol{\alpha})^{m} v}{(1+v)(1-2 v)} \varepsilon_{i i} \varepsilon_{k k}+\frac{\sum_{m=0}^{p} a_{m} \mathcal{J}_{2}(\boldsymbol{\alpha})^{m}}{2(1+v)}\left[\varepsilon_{i j} \varepsilon_{i j}+\varepsilon_{i j} \varepsilon_{j i}\right]
$$

Since $\boldsymbol{\varepsilon}_{\mathbf{v}}$ is a symmetric tensor, this expression leads to

$$
\frac{1}{2} \boldsymbol{\varepsilon}_{\mathbf{v}}: \mathbb{C}_{\mathbf{v}}(\boldsymbol{\alpha}): \boldsymbol{\varepsilon}_{\mathbf{v}}=\frac{\sum_{m=0}^{p} a_{m} \mathcal{J}_{2}(\boldsymbol{\alpha})^{m} v}{2(1+v)(1-2 v)} \operatorname{tr}\left(\boldsymbol{\varepsilon}_{\mathbf{v}}\right)^{2}+\frac{\sum_{m=0}^{p} a_{m} \mathcal{J}_{2}(\boldsymbol{\alpha})^{m}}{2(1+v)} \boldsymbol{\varepsilon}_{\mathbf{v}}: \boldsymbol{\varepsilon}_{\mathbf{v}}
$$

or

$$
\frac{1}{2} \boldsymbol{\varepsilon}_{\mathbf{v}}: \mathbb{C}_{\mathbf{v}}(\boldsymbol{\alpha}): \boldsymbol{\varepsilon}_{\mathbf{v}}=\underbrace{\left(\frac{v}{2(1+v)(1-2 v)} \operatorname{tr}\left(\boldsymbol{\varepsilon}_{\mathbf{v}}\right)^{2}+\frac{1}{2(1+v)} \boldsymbol{\varepsilon}_{\mathbf{v}}: \boldsymbol{\varepsilon}_{\mathbf{v}}\right)}_{A} \sum_{m=0}^{p} a_{m} \mathcal{J}_{2}(\boldsymbol{\alpha})^{m}
$$

Therefore, the expression 37 becomes

$$
\frac{\partial}{\partial \boldsymbol{\alpha}}\left[\frac{1}{2} \boldsymbol{\varepsilon}_{\mathbf{v}}: \mathbb{C}_{\mathbf{v}}(\boldsymbol{\alpha}): \boldsymbol{\varepsilon}_{\mathbf{V}}\right]=\frac{\partial}{\partial \mathcal{J}_{2}}\left[A \sum_{m=0}^{p} a_{m} \mathcal{J}_{2}(\boldsymbol{\alpha})^{m}\right] \frac{\partial \mathcal{J}_{2}}{\partial \boldsymbol{\alpha}}
$$

As the first term is trivial and the second is classic, we have finally

$$
\frac{\partial}{\partial \boldsymbol{\alpha}}\left[\frac{1}{2} \boldsymbol{\varepsilon}_{\mathbf{v}}: \mathbb{C}_{\mathbf{v}}(\boldsymbol{\alpha}): \boldsymbol{\varepsilon}_{\mathbf{v}}\right]=\frac{3}{2}\left[\frac{v}{(1+v)(1-2 v)} \operatorname{tr}\left(\boldsymbol{\varepsilon}_{\mathbf{v}}\right)^{2}+\frac{1}{(1+v)} \boldsymbol{\varepsilon}_{\mathbf{v}}: \boldsymbol{\varepsilon}_{\mathbf{v}}\right] \sum_{m=1}^{p} m a_{m} \mathcal{J}_{2}(\boldsymbol{\alpha})^{m-2} \boldsymbol{\alpha}
$$

As the viscous strain tensor is supposed deviatoric here, the last equation can be simplified

$$
\frac{\partial}{\partial \boldsymbol{\alpha}}\left[\frac{1}{2} \boldsymbol{\varepsilon}_{\mathbf{v}}: \mathbb{C}_{\mathbf{v}}(\boldsymbol{\alpha}): \boldsymbol{\varepsilon}_{\mathbf{v}}\right]=\frac{3}{2} \frac{1}{(1+v)} \boldsymbol{\varepsilon}_{\mathbf{v}}: \boldsymbol{\varepsilon}_{\mathbf{v}} \sum_{m=1}^{p} m a_{m} \mathcal{J}_{2}(\boldsymbol{\alpha})^{m-2}
$$

Taking into account the typical values of the constitutive parameters (see "Appendix B"), it can be shown that

$$
\left|\frac{3}{2} \frac{1}{(1+v)} \boldsymbol{\varepsilon}_{\mathbf{v}}: \boldsymbol{\varepsilon}_{\mathbf{v}} \sum_{m=1}^{p} m a_{m} \mathcal{J}_{2}(\boldsymbol{\alpha})^{m-2}\right| \ll \frac{2}{3} C
$$

thus leading to the simplification made in Eq. 8. Some additional elements are given in "Appendix B".

\section{B Numerical validation of the simplification of the state equation relative to $X$}

We propose in this appendix to check numerically the simplification that has been proposed in Sect. 4, Eq. 8 and demonstrated in "Appendix A". The approach consists in considering the typical order of magnitude of the quantities taking into account the identified set of parameters (see Table 1) considering an uniaxial stress state. The generalization to any stress state is left to the reader. 
In the case of an uniaxial stress state, we have (see Sect. 4.2)

$$
\begin{gathered}
\boldsymbol{X}(t)=X \mathbf{e}_{1} \otimes \mathbf{e}_{1}-\frac{X}{2}\left(\mathbf{e}_{2} \otimes \mathbf{e}_{2}+\mathbf{e}_{3} \otimes \mathbf{e}_{3}\right) \\
\boldsymbol{\alpha}(t)=\alpha \mathbf{e}_{1} \otimes \mathbf{e}_{1}-\frac{\alpha}{2}\left(\mathbf{e}_{2} \otimes \mathbf{e}_{2}+\mathbf{e}_{3} \otimes \mathbf{e}_{3}\right) \\
\boldsymbol{\varepsilon}_{\mathbf{p}}=\varepsilon_{\mathrm{p}} \mathbf{e}_{1} \otimes \mathbf{e}_{1}-\frac{\varepsilon_{\mathrm{p}}}{2}\left(\mathbf{e}_{2} \otimes \mathbf{e}_{2}+\mathbf{e}_{3} \otimes \mathbf{e}_{3}\right) \\
\boldsymbol{\varepsilon}_{\mathbf{v}}=\varepsilon_{\mathrm{v}} \mathbf{e}_{1} \otimes \mathbf{e}_{1}-\frac{\varepsilon_{\mathrm{v}}}{2}\left(\mathbf{e}_{2} \otimes \mathbf{e}_{2}+\mathbf{e}_{3} \otimes \mathbf{e}_{3}\right)
\end{gathered}
$$

Since $\gamma=0$, the Armstrong-Fredericks nonlinear kinematic hardening is reduced to a linear Prager model. Therefore,

$$
X=C \varepsilon_{\mathrm{p}}
$$

and

$$
\alpha=-\frac{3}{2} \frac{1}{C} X=-\frac{3}{2} \varepsilon_{\mathrm{p}}
$$

thus

$$
\mathcal{J}_{2}(\boldsymbol{\alpha})=\frac{3}{4} \varepsilon_{\mathrm{p}}
$$

We also have

$$
\boldsymbol{\varepsilon}_{\mathbf{v}}: \boldsymbol{\varepsilon}_{\mathbf{v}}=\frac{3}{2} \varepsilon_{\mathrm{v}}^{2}
$$

Equation 52 becomes

$$
\left|\frac{2 a_{1}}{(1+v)} \frac{\varepsilon_{\mathrm{v}}^{2}}{\varepsilon_{\mathrm{p}}}\right| \ll \frac{2}{3} C
$$

Considering the set of parameters (Table 1) and typical values for the viscoelastic strain $\left(10^{-3}\right)$, the plastic strain $\left(10^{-3}\right)$ and the Poisson coefficient (0.4), the previous equation gives

$$
\left|\frac{2}{(1+v)} \varepsilon_{\mathrm{v}}^{2} a_{1} \frac{1}{\varepsilon_{\mathrm{p}}}\right| \ll \frac{2}{3} C
$$

or

$$
28 \ll 156000
$$

The simplification proposed in Eq. 8 is therefore fully justified.

\section{References}

1. Advani, S., Tucker, C.: A numerical simulation of short fiber orientation in compression molding. Polym. Compos. 11(3), 164-173 (1990)

2. Andriyana, A., Billon, N., Silva, L.: Mechanical response of a short fiber-reinforced thermoplastic: experimental investigation and continuum mechanical loading. Eur. J. Mech. A 29, 1065-1077 (2010)

3. Benoit, A., Maitournam, M., Rémy, L., Oger, F.: Cyclic behaviour of structures under thermomechanical loadings: application to exhaust manifolds. Int. J. Fatigue 38, 65-74 (2012)

4. Bernasconi, A., Cosmi, F., Hine, P.: Analysis of fibre orientation distribution in short fibre reinforced polymers: a comparison between optical and tomographic methods. Compos. Sci. Technol. 72, 2002-2008 (2012)

5. Blaber, J., Adair, B., Antoniou, A.: Ncorr: open-source 2D digital image correlation matlab software. Exp. Mech. 55, 1105$1122(2015)$

6. Chaboche, J.: Thermodynamic formulation of constitutive equations and application to the viscoplasticity and viscoelasticity of metals and polymers. Int. J. Solids Struct. 34, 2239-2254 (1997)

7. Colak, O.: Modeling deformation behavior of polymers with viscoplasticity theory based on overstress. Int. J. Plast. 21, 145-160 (2005)

8. Constantinescu, A., Van Dang, K., Maitournam, M.: A unified approach for high and low cycle fatigue based on shakedown concepts. Fatigue Fract. Eng. Mater. Struct. 26, 561-568 (2003)

9. Dray Bensahkoun, D.: Pédiction des propriétés thermo-élastiques d'un composite injecté et chargé de fibres courtes. Ph.D. Thesis, ENSAM Paris (2006)

10. Drozdov, A., Dusunceli, N.: Cyclic deformations of polypropylene with a strain-controlled program. Polym. Eng. Sci. 52, 2316-2326 (2012)

11. Halphen, B., Nguyen, Q.: Sur les matériaux standards généralisés. J. Mec. 14, 39-63 (1975) 
12. Jégou, L., Marco, Y., Le Saux, V., Calloch, S.: Fast prediction of the wöhler curve from heat build-up measurements on short fiber reinforced thermoplastics. Int. J. Fatigue 47, 259-267 (2012)

13. Kichenin, J.: Comportement thermomécanique du polyéthylène. Application aux structures gazières. Ph.D. Thesis, Ecole Polytechnique (1992)

14. Klimkeit, B., Nadot, Y., Castagnet, S., Nadot-Martin, C., Dumas, C., Bergamo, S., Sonsino, C., Buter, A.: Multiaxial fatigue life assessment for reinforced polymers. Int. J. Fatigue 33, 766-780 (2011)

15. Krairi, A., Doghri, I.: A thermodynamically-based constitutive model for thermoplastic polymers coupling viscoelasticity, viscoplasticity and ductile damage. Int. J. Plast. 60, 163-181 (2014)

16. Krairi, A., Doghri, I., Robert, G.: Multiscale high cycle fatigue models for neat and short fiber reinforced thermoplastic polymers. Int. J. Fatigue 92, 179-192 (2016)

17. Krairi, A.: Multiscale modeling of the damage and failure of homogeneous and short-fiber reinforced thermoplastics under monotonic and fatigue loadings. Ph.D. Thesis, Université Catholique de Louvain (2015)

18. Launay, A., Maitournam, M., Marco, Y., Raoult, I.: Multiaxial fatigue models for short glass fiber reinforced polyamide. Part II: fatigue life estimation. Int. J. Fatigue 47, 390-406 (2013)

19. Launay, A., Maitournam, M., Marco, Y., Raoult, I., Szmytka, F.: Cyclic behaviour of short glass fibre reinforced polyamide: experimental study and constitutive equations. Int. J. Plast. 27, 1267-1293 (2011)

20. Launay, A., Marco, Y., Maitournam, M., Raoult, I.: Modelling the influence of temperature and relative humidity on the time-dependent mechanical behaviour of a short glass fibre reinforced polyamide. Mech. Mater. 56, 1-10 (2013)

21. Le Saux, V., Doudard, C.: Proposition of a compensated pixelwise calibration for photonic infrared cameras and comparison to classic calibration procedures: case of thermoelastic stress analysis. Infrared Phys. Technol. 80, 83-92 (2017)

22. Lemaitre, J., Chaboche, J.: Mechanics of Solid Materials. Cambridge University Press, Cambridge (1990)

23. Leveuf, L., Marco, Y., Le Saux, V., Navrátil, L., Leclercq, S.: Fast screening of the fatigue properties of thermoplastics reinforced with short carbon fibers based on thermal measurements. Polym. Test. (2017) (submitted)

24. Marco, Y., Le Saux, V., Jégou, L., Launay, A., Serrano, L., Raoult, I., Calloch, S.: Dissipation analysis in SFRP structural samples: thermomechanical analysis and comparison to numerical simulations. Int. J. Fatigue 67, 142-150 (2014)

25. Marco, Y.: Caractérisation multi-axiale du comportement et de la micro-structure d'un semi-cristallin: application au cas du P.E.T. Ph.D. Thesis, Ecole Normale Supérieure de Cachan (2003)

26. Masquelier, I., Marco, Y., Le Saux, V., Calloch, S., Charrier, P.: Determination of dissipated energy fields from temperature mappings on a rubber-like structural sample: experiments and comparison to numerical simulations. Mech. Mater. 80, 113$123(2015)$

27. Mortazavian, S., Fatemi, A.: Fatigue of short fiber thermoplastic composites: a review of recent experimental results and analysis. Int. J. Fatigue 102, 171-183 (2017)

28. Ostwald, W.: Ueber die rechnerische darstellung des strukturgebietes der viskosität. Kolloid Z. 47, $176-187$ (1929)

29. Praud, F., Chatzigeorgiou, G., Bikard, J., Meraghni, F.: Phenomenological multi-mechanisms constitutive modelling for thermoplastic polymers, implicit implementation and experimental validation. Mech. Mater. 114, 9-29 (2017)

30. Rémond, Y.: Constitutive modelling of viscoelastic unloading of short glass fibre-reinforced polyethylene. Compos. Sci. Technol. 65, 421-428 (2005)

31. Selmi, A., Doghri, I., Adam, L.: Micromechanical simulations of biaxial yield, hardening and plastic flow in short glass fiber reinforced polyamide. Int. J. Mech. Sci. 53, 696-706 (2011)

32. Serrano, L., Marco, Y., Le Saux, V., Robert, G., Charrier, P.: Fast prediction of the fatigue behavior of short-fiber-reinforced thermoplastics based on heat build-up measurements: application to heterogeneous cases. Contin. Mech. Thermodyn. 29, $1113-1133(2017)$

33. Vincent, M., Giroud, T., Clarker, A.: Eberhardt: description and modeling of fiber orientation in injection molding of fiber reinforced thermoplastics. Polymer 46, 6719-6725 (2005) 